\title{
Erratum to: Flow shops with WIP and value added costs
}

\author{
Jaehwan Yang • Marc E. Posner
}

Published online: 15 February 2015

(C) Springer Science+Business Media New York 2015

\section{Erratum to: J Sched (2010) 13:3-16}

\section{DOI 10.1007/s10951-009-0130-z}

We correct four errors in our paper. The first is that the equation

$W_{j}=\sum_{i=1}^{m}\left(w_{2 i-1} Q_{2 i-1, j}+w_{2 i} p_{2 i, j}\right)$

on Page 5 in Sect. 2 should read

$W_{j}=\sum_{i=1}^{m}\left(w_{2 i-1} Q_{i j}+w_{2 i} p_{i j}\right)$.

The next error concerns Lemma 2. As stated, it is incorrect. Hatem Hadda noticed that the lemma holds when there are only two machines. We observe that it also holds for the $m$ machine case by changing the statement slightly to:

Lemma 2 For the problem $F \| \sum W_{j}$, suppose that $p_{i j} \geq$ $p_{i+1, j}$ for all $i \in M \backslash\{m\}$ and $j \in N$. Also, suppose that $\sum_{i=1}^{k} p_{i j} \leq \sum_{i=1}^{k} p_{i, j+1}$ for all $k \in M$ and $j \in N \backslash\{n\}$. Then, scheduling the jobs without inserted idle time in the order $(1,2, \ldots, n)$ is optimal.

The final errors are in the statement of Lemma 3, which contains two typos. The first is that $[j]$ should mean the job

The online version of the original article can be found under doi:10.1007/s10951-009-0130-z.

\section{J. Yang}

School of Business Administration, University of Seoul,

13 Siripdae-gil, Dongdaemun-gu, Seoul 130-743, Korea

M. E. Posner (凶)

Department of Integrated Systems Engineering,

The Ohio State University, Columbus, OH, USA

e-mail: posner.1@osu.edu in position $j$ on Machine $l($ not Machine $m)$. The second is that the first term after the inequality

$w_{1} n \sum_{i=1}^{l-1} p_{i[l]}$

should be

$w_{1} n \sum_{i=1}^{l-1} p_{i[1]}$.

Acknowledgments We thank Hatem Hadda for noticing the errors in our paper. 